Original manuscript Australia.

\title{
Bone accrual over 18 months of participation in different loading sports
} during childhood and adolescence \section{Runing title: Bone health and sport participation} Ricardo Ribeiro Agostinete ${ }^{1}$, Dimitris Vlachopoulos ${ }^{2}$, André Oliveira Werneck ${ }^{1}$, Santiago Maillane Vanegas ${ }^{1}$, Kyle Robinson Lynch ${ }^{1}$, Geraldine Naughton ${ }^{3}$, Romulo Araújo Fernandes ${ }^{1}$

1- Laboratory of InVestigation in Exercise (LIVE), Department of Physical Education, Sao Paulo State University (UNESP), Presidente Prudente, Brazil.

2- Children's Health and Exercise Research Centre, Sport and Health Sciences, University of Exeter, United Kingdom.

3- School of Exercise Science, Australian Catholic University, Fitzroy, VIC,

Funding Sources and Financial Disclosure: This study was supported by the São Paulo Research Foundation-FAPESP (Process 2013/06963-5, 2015/13543-8, 2016/06920-2, 2017/09182-5 and 2018/24164-6). AOW received a Grant from the FAPESP (2017/27234-2). SMJ received a Grant from the FAPESP (2016/20354-0) and KRL received a Grant from the FAPESP (2016/20377-0).

26 Conflict of interest: Ricardo Agostinete, Dimitris Vlachopoulos, André Werneck, 27 Santiago Vanegas, Kyle Lynch, Geraldine Naughton, Romulo Fernandes declare that they 8 have no conflict of interest.

Acknowledgements: Sao Paulo Research Foundation (FAPESP) and the effort of the 1 participants and their parents and coaches.

* Corresponding author: Ricardo Ribeiro Agostinete (ricardo.agostinete@ unesp.br) 305 Roberto Simonsen - Presidente Prudente, SP, Brazil. 19060 -900 
Bone accrual over 18 months of participation in different loading sports during childhood and adolescence

Mini-abstract

This study investigated the association of impact and non-impact sports on bone mineral density accrual in adolescents for 18 months. The impact sports were beneficial for the

42 bone health (accrual of bone density), on the other hand, swimmers had similar and lower 43 bone mineral density compared to the control group.

Abstract

Purpose: To investigate the association of impact and non-impact sports on bone mineral density (BMD) accrual in adolescents of both sexes over a period of 18 months. Methods: The sample was composed of 71 children and adolescents, aged 9 to 17 years at baseline. Bone outcomes were compared according to sports (impact, $n=33$ [basketball, karate, and

50 judo], non-impact, $n=18$ [swimming], and control group, $n=20$ ). aBMD was measured by 51 Dual-energy X-ray absorptiometry (DXA) and bone mineral apparent density (BMAD) estimated through equation. The statistics analysis was composed by Analysis of variance (ANOVA and ANOVA repeated measures). Results: Adjusted aBMD at lower limbs, whole body less head (WBLH) and adjusted BMAD at WBLH were significantly greater in the impact sports group than the non-impact sport group at all time points, besides in 9 and 18 months in upper limbs and total spine at baseline. Non-impact sports group also presented lower values of aBMD compared to control group in lower limbs and WBLH at 9-months and at 9 and 18-months in BMAD WBLH. There was a significant interaction (time $\mathrm{x}$ sports group) at upper limbs $(\mathrm{p}=0.042)$, WBLH $(\mathrm{p}=0.006)$ aBMD and BMAD WBLH $(\mathrm{p}<0.001)$. Conclusion: Overall, impact sports were more beneficial on accumulating BMD over a period of 18 months, while swimmers had similar and lower BMD compared to the control group.

63

64 Keywords: Bone development; Longitudinal; Physical activity; Sports training Youth; Bone density. 


\section{Introduction}

The first two decades of life seem to be extremely important for bone acquisition for bone acquisition. According to the literature, around $50 \%$ of whole body bone mineral content (BMC) is acquired during childhood and adolescence [1], especially among from the years surrounding peak height velocity [2]. Increased gains in bone mineral density (BMD) during adolescence may decrease the risk of developing osteoporosis [3] by achieving the full potential of peak bone mass.

The growth of bone tissues is largely determined by non-modifiable factors, such as genetics and hormones $[4,5]$. However, environmental factors, such as physical activity and nutrition, can alter BMC and BMD during growth [6-8]. The positive effects of physical activity on bone health can be supported by an increased action of anabolic hormones and proteins, such as growth hormone $(\mathrm{GH})$ and insulin-like growth factor 1 (IGF-1) $[9,10]$. Moreover, physical activity can induce positive bone adaptations via muscle contractions performed during movements that can generate strain and stimulate bone matrix by modifying geometry and strength of trabecular and cortical bones according to muscle-bone unit theory $[10,11]$

Among the different manifestations of physical activity, sports participation offers numerous physiological benefits [12] and accounts for a large part of physical activity

84 during childhood and adolescence worldwide [13]. Sports can be considered as impact and non-impact based on the review developed by Tenforde \& Fredericson (2011) [10], which characterizes modalities of impact, those that involve ground-reaction forces. In contrast, non-impact modalities are performed in "hypogravity" environments, without ground-reaction forces. 
The loading of skeleton during specific sport practice is a key component of the

90 bone adaptations, but the magnitude of the stimuli depends on the training environment

91 (e.g., hard surface, such as basketball, or hypogravity environment, such as swimming)

$92[14,15]$, the frequency (e.g., sessions and days per week), intensity (e.g., light, moderate,

93 vigorous) and volume (e.g., sets, repetitions) of each sport [16]. However, a recent review

94 by Bielemaan et al. (2013) indicated the lack of a consensus on which type of exercise

95 performed in early life can most effectively promote bone health [17]. Considering the

96 amount of bone mass acquired during childhood and adolescence [2], and that the

97 majority of children and adolescence engaged in different types of sports, it is of great

98 interest to determine the long-term association of different loading sports and bone

99 acquisition during this period of life.

100 Based on the absence of consensus about the role of different sports and bone

101 growth, cross-sectional studies have sought to identify the association between different

102 types of sports, such as volleyball [18], football [7], basketball [19], tennis [20], martial

103 arts [21] and bone outcomes compared to controls and other sports. Longitudinal studies

104 enable researchers to assess more appropriately causality than cross-sectional studies, but

105 there are only a few longitudinal studies focusing on the association of different loading

106 sports and bone development $[22,23]$. Most of studies in the literature analyzing the

107 longitudinal impact of sports participation in bone density of adolescents were developed

108 up to 12-month of follow-up [24-26]. In fact, a smaller number of studies followed

109 adolescents over 12 months, but those studies have focused in comparisons based on "one

110 single sport versus control group", while comparisons according to weigh-bearing

111 characteristics (impact, non-impact sports and control group) were not presented by them

112 [27-29]. These studies also did not ponder a volumetric interpretation of BMD 
113 considering the influence of body size, as proposed in the use of bone mineral apparent

114 density (BMAD) [30]. Therefore, it is important to understand more comprehensively the

115 long-term relation of sports participation in different levels of ground reaction force and

116 bone accrual during adolescence.

117 The purpose of this longitudinal study was to compare the long-term of impact

118 (basketball, karate, and judo) and non-impact (swimming) sports on aBMD and BMAD

119 accrual in children and adolescents over a period of 18 months. It was hypothesized that

120 impact sports will have greater bone accrual compared to non-impact sports.

\section{Methods}

\section{Study design}

123 This longitudinal study is part of a large cohort study that was conducted in the

124 city of Presidente Prudente from October 2013 to May 2015. The study was approved by

125 the Ethical Research Committee of the São Paulo State University-UNESP (Process

126 number 02891112.6.0000.5402).

127 At the beginning of the study in 2013, a cooperation agreement was formed

128 between the Laboratory of InVestigation in Exercise-LIVE and the municipal secretariat

129 of Education and Sports of Presidente Prudente. After explaining the proposal to school

130 principals and sports coaches, the researchers were given formal authorization to start

131 data collection. Children and adolescents not actively engaged in any sport and who were

132 enrolled in the schools of the Secretary of Education were invited to join such as the

133 control group. The athletic group comprised adolescents of the modalities representing

134 their municipality: judo, karate, basketball, and swimming. To participate in the study,

135 adolescents were required to provide informed consent signed by a parent or guardian.

\section{Participants}


The inclusion criteria to be eligible were: (1) chronological age between 9 and 17

138 years at baseline; (2) no current use of medication that could affect bone metabolism, and

139 (3) regular participation in school physical education classes (two hours per week). For

140 sports group: (1) a minimum of 6 months of participation in the specific sport (considering

141 previous studies) [31, 32]; (2) non-involvement in other sports. For control group: (1) the

142 absence of previous engagement in organized and supervised sports before baseline

143 measures (3 months) and during the 18 months of the study.

144 At baseline, 184 children and adolescents (108 males and 76 females) of both

145 sexes were recruited, after 9 months 126 adolescents (78 males and 48 females)

146 completed the second visit and 71 (42 males and 29 females) children and adolescents

147 completed the third visit over 18 months. The drop out of 113 children and adolescents

148 was mainly due to disinterest in continuing as a volunteer in the study, absence in the

149 scheduled measurement, and moving to another city in control group and interrupting

150 sports participation or being transferred to another team in sports group. The present study

151 included only valid data from the 3-time points (no missing data). Potential selection bias

152 (71 children and adolescents who remained versus those 113 children and adolescents

153 who dropout) was assessed through the differences at baseline. The groups were similar

154 to chronological age $(\mathrm{t}$ test; $\mathrm{p}$-value $=0.126)$, body mass $(\mathrm{t}$ test; $\mathrm{p}$-value $=0.072)$, height

155 ( $\mathrm{t}$ test; $\mathrm{p}$-value $=0.112)$, years from PHV ( $\mathrm{t}$ test; $\mathrm{p}$-value $=0.097)$, age of PHV $(\mathrm{t}$ test;

$156 \mathrm{p}=$ value $=0.983)$, fat mass $(\mathrm{t}$ test; $\mathrm{p}$-value $=0.335)$, lean soft tissue $(\mathrm{t}$ test; $\mathrm{p}$-value $=$

157 0.322), upper limbs aBMD ( $\mathrm{t}$ test; $\mathrm{p}$-value $=0.272)$, lower limbs aBMD ( test; $\mathrm{p}$-value $=$

158 0.230), total spine aBMD ( $\mathrm{t}$ test; $\mathrm{p}$-value $=0.150)$ and whole body aBMD ( $\mathrm{t}$ test; $\mathrm{p}$-value

$159=0.124)$. Lastly, the drop out predominantly occurred in control group (76.5\%) compared

160 to non-impact $(40.0 \%)$ and impact sports $(52.2 \%)($ chi-squared with p value $=<0.001)$. 
162 group comprised 20 children and adolescents (12 males and 8 females). Sports were

163 categorized according to potential osteogenic impact. The impact group comprised 33

164 children and adolescents: basketball (8 males) and martial arts (11 males and 14 females

165 in karate and judo). Finally, the non-impact group comprised 18 adolescent swimmers

166 (11 males and 7 females) (figure 1). Athletes from karate and swimming participated in

167 competitions at the national level and the basketball players participated in state

168 tournaments. The remaining athletes (judo) competed at the regional level.

169

170 Previous Sports Participation and Training Routine

171 The athletes reported the time (in months) of practice. Similarly, coaches provided

172 information about the volume and frequency of training (minutes trained daily and the

173 number of days trained in a week, respectively). Exposure to resistance training was also

174 consulted with coaches and athletes and considered as a confounding factor.

175

176 Anthropometry

177 Stature and sitting height were measured using a stadiometer (Sanny, model

178 American Medical of the Brazil Ltda, Brazil, accurate to $0.1 \mathrm{~cm}$ ) that permitted an

179 estimate of maturity offset. The body mass was measured using an electronic scale

180 (Filizzola PL 150, model Filizzola Ltda, Brazil with a precision of $0.1 \mathrm{~kg}$ ). All measures

181 were assessed using standardized techniques by a single trained researcher. The technical

182 errors of measurement were $0.041 \%, 0.110 \%$ and $0.157 \%$ for body mass, height, and 183 sitting-height, respectively.

184

185 Body Composition and Bone Mineral Density 
Areal Bone mineral density (aBMD, $\left.\mathrm{g} / \mathrm{cm}^{2}\right)$, lean soft tissue (LST, $\mathrm{kg}$ ) and fat mass (FM, kg) were measured at the university laboratory in a temperature-controlled room using dual-energy x-ray absorptiometry (Lunar DPX-NT; General Electric

190 software (version 4.7). A trained researcher performed all scans and tested the scanner

191 quality before the first exam of each day. The coefficient of variation for this device was

$1920.66 \%$ (in whole body BMD analysis, $n=30$ participants not involved in this study). The scans were performed using a standardized protocol with the participants remaining in the supine position and wearing only light clothing, without shoes. Regional analysis of BMD in upper limbs, lower limbs, total spine (cervical, thoracic and lumbar) and WBLH occurred off-line after the scans took place [33] following the recommendations of manufacturer recommendations and previous studies [34, 35]: defined comprising all soft tissues (the same for right and left sides).

-Lower Limbs: measured considering the position of the line passing through on the lower edge of the ischium and the lateral and medial lines defined comprising all soft tissues (the same for right and left sides). level) to the lower edge of the chin. The lateral cut positioned as close as possible to the 206 spine.

The bone mineral apparent density $\left(\mathrm{BMAD}-\mathrm{g} / \mathrm{cm}^{3}\right)$ was calculated by the equation: $\mathrm{BMAD}_{\mathrm{TB}}=\mathrm{BMC} /\left(\right.$ total body $\mathrm{BA}^{2} /$ body height $)$ proposed by Katzman et al. 
210 (BMAD) by dividing bone mineral content (BMC) by the three-dimensional bone volume

211 derived from its two-dimensional projected bone area (BA).

212

213

\section{Somatic Maturation}

Anthropometric measurements (body mass, stature, and sitting height) were used

216 to calculate years from the age of peak height velocity (PHV) through mathematical

217 formulas predicted by Moore et al. [36]. This measure denotes the time remaining (years)

to reach the age of PHV. Finally, by subtracting chronological age by years from the peak

height velocity, it is possible to find the age of the peak height velocity (APHV).

Years from age of PHV for males $=-8.128741+[0.0070346 *($ Age $*$ Sitting

222

223

224

225

226

227

228

229

230

231

232

233

Height)]

Years from age of PHV for females $=-7.709133+[0.0042232 *($ Age $*$ Stature $)]$ Age of $\mathrm{PHV}=$ Chronological age - years from age at PHV.

$\mathrm{R}^{2}=0.896(\mathrm{SEE}+0.542)$ in boys and $\mathrm{R}^{2}=0.898(\mathrm{SEE}+0.528)$ in girls .

\section{Consumption of vitamin D}

Utilizing a questionnaire about foods rich in vitamin D (commonly observed in a Brazilian diet) made by a nutritionist. The adolescents reported the frequency of consumption (Likert scale) during the previous week the evaluations. The sum of the generated score was considered proxy of vitamin D intake and inserted in the analysis as utilized in previous studies[24, 31]. 


\section{Statistical Analyses}

The sample was calculated considering bone density gains (sport participant

238 versus non-sport participant) of $0.05 \mathrm{~g} / \mathrm{cm}^{2}$ [24], considering a standard deviation of 0.07

$239 \mathrm{~g} / \mathrm{cm}^{2}, 80 \%$ power and $5 \%$ error-alpha. Taking into account all these parameters, the

240 minimum sample size was estimated in 15 children and adolescents in each group.

241 Following checks for data distribution, the descriptive analyses were reported in 242 mean and standard deviation (SD), as well as appropriate $95 \%$ confidence levels. Analysis

243 of Variance - ANOVA was performed for initial comparisons between groups using 244 Bonferroni post-hoc test. ANOVA- repeated measures (3 groups x 3 time points) was 245 used for comparisons in aBMD accrual at different skeletal sites after adjustment for 246 APHV, LST of the specific site, volume of training, practice of resistance training 247 (Categorical variable [yes or no]), sporting experience (months), fat mass, height, vitamin 248 D intake and sex $[23,37]$ and the differences among groups observed through confidence 249 interval. The statistical analyses were performed using BioEstat (version 5.0) and the 250 significance level was set at $\mathrm{p}$-value $<0.05$.

\section{$251 \quad$ Results}

Table 1 shows participants' descriptive characteristics at baseline by sport. Seventy-one children and adolescents (42 males and 29 females) with valid data on the three-time points were included. Control group showed significantly lower values for height $(\mathrm{p}=0.041)$ and LST $(\mathrm{p}=0.001)$ than the non-impact group and lower LST also compared to the impact group $(\mathrm{p}=0.014)$. In addition, the non-impact group presented a higher volume of training than the impact group (weekly training) $(\mathrm{p}=0.001)$. Comparisons of aBMD showed similar values among all groups at the total spine. However, the impact group had significantly higher aBMD at the upper limbs $(\mathrm{p}=0.033)$, 
260 lower limbs $(\mathrm{p}=0.019)$ and WBLH $(\mathrm{p}=0.019)$ than the control group (table 1). Lastly, non-impact sports showed lower BMAD of WBLH than impact sports $(\mathrm{p}=0.038)$ and also control group $(\mathrm{p}=0.027)$.

The non-impact group presented significantly lower values of aBMD at the upper

limbs (Figure 2, Panel A), compared to the impact group at 9 months (mean of 0.751 in non-impact sports vs. 0.828 in impact sports) and 18 months (mean of 0.782 in nonimpact sports vs. 0.869 in impact sports). Furthermore, the control group presented lower of aBMD at the upper limbs compared to the impact group in baseline (mean of 0.699 in control group vs. 0.770 in impact sports) and 18 months (mean of 0.795 in control group vs. 0.869 in impact sports).

Furthermore, there were significant differences in aBMD at lower limbs between groups (Figure 2, Panel B), the non-impact group had significantly lower values compared to the impact group in the three moments (mean of 1.063 vs. 1.179 at baseline; mean of 1.112 vs. 1.234 in 9 months and 1.153 vs. 1.295 in 18 months in non-impact and impact sports, respectively) and lower values compared to the control group in 9-months (mean of 1.112 in non-impact vs. 1.226 in control group). Regarding aBMD in total spine, the impact group presented significantly higher values compared to the non-impact group at baseline (mean of 0.891 in non-impact vs. 0.974 in impact sports) (Figure 2, Panel C). WBLH when compared to both the control group and impact sports at 9 months (mean of 2800.935 in non-impact vs. 1.037 in impact sports and 1.037 for control group) and with the 281 impact group at baseline (0.908 in non-impact vs. 0.992 in impact sports) and 18-months 282 (0.977 in non-impact sports vs. 1.096 in impact sports) (Figure 2, Panel D). There was a significant interaction between "time*sport" at the upper limbs $(p=0.042)$ and the WBLH $(\mathrm{p}=0.006)$ aBMD. The sport practiced significantly affected all bone variables 
285 (upper limbs, $\mathrm{p}=0.001$, lower limbs, $\mathrm{p}=0.001$; total spine, $\mathrm{p}=0.013$; WBLH, $\mathrm{p}=0.001$ ) 286 over the 18-month studied period.

287 When analyzing BMAD of whole body, more expressive differences were 288 observed between the groups. The non-impact group showed significant lower values 289 compared to the impact group and control group in 9 months (mean of 0.081 in non290 impact sport vs. 0.094 in control group and 0.091 for impact sports) and 18 months (mean 291 of 0.081 vs. 0.091 and 0.031 for non-impact, control group and impact group, respectively) and the impact group at baseline (mean of 0.085 in non-impact sport vs. 0.093 in impact group) (Figure 3). The time $(\mathrm{p}=0.002)$ and sport practiced $(\mathrm{p}<0.001)$

294 significantly affected the BMAD, as well there was a significant interaction between 295 "time*sport $(\mathrm{p}<0.001)$.

\section{Discussion}

The main findings of the present study indicate that children and adolescents involved in impact sports, such as basketball and marital arts, over a period of 18-months

300 have significantly higher adjusted aBMD and BMAD accrual compared to non-impact 301 sports, such as swimming. Furthermore, swimmers also have a significantly lower aBMD and BMAD accrual at the WBLH and lower limbs compared to the control group. of each sport $[11,25] ; 2)$ the different forces continuously applied to the skeleton over a 305 long period of time $[10,38,39]$.

306 Understanding the association of sports participation and bone health during 307 adolescence has been debated in the literature [24, 40, 41]. The findings of the present study show that participation in impact sports, such as basketball and martial arts, affects differently the aBMD and BMAD accrual of which they found to have higher aBMD and 
310 BMAD compared to adolescents practicing non-impact sports, such as swimming. These

311 results confirmed our hypothesis that the impact sports are beneficial for aBMD accrual

312 [25]. Prolonged exposure in impact sports can increase the mechanical loading on the

313 bone matrix. Sports modalities, such as basketball and martial arts (modalities of our

314 study) involve movements, such as jumps, sprints, changes of direction, high impact and

315 start stops. These movements generate compressions and tensions in the bones [11, 39]

316 generating a process of metabolic stress and consequently stimulating bone formation $317[10,38]$.

318 A study of Zribi et al assessed bone outcomes in prepubescent adolescent 319 basketball athletes aged 11.1 years compared to controls [19]. It was found that basketball 320 players had significantly higher BMC at upper, lower limbs and WBLH than controls, 321 which is in accordance with the results of the current study. A different study of Ito et al 322 showed that 9-months of judo participation improved significantly aBMD accrual 323 compared to controls in males aged 12.9 years [42]. Furthermore, previous evidence 324 shows that bone adaptations are site-specific, according to the skeletal sites stimulated by 325 the sport specific patterns [43]. Previous studies indicate that the skeletal sites most 326 benefited by the practice of the specific sport are those directly stimulated, such as legs 327 aBMD in basketball players [24], arms BMD for judo fighters [21], dominant foot in 328 adolescent footballers [7] and dominant arm in tennis players [20].

329 Results from the current study are also supported by a recent cross-sectional study 330 involving adolescent football, swimming and cycling adolescent male athletes [44]. The 331 study concluded that adolescent males involved in weight-bearing sports, such as football, 332 had higher bone mass, bone geometry, and bone stiffness than those involved in non333 weight bearing sports, such as swimming and cycling. The same study found that 334 swimming and cycling adolescent athletes presented similar bone status with an active 
335 control group, and the authors called for more longitudinal studies to understand the 336 association of the 'non-osteogenic' sports participation and bone accrual during growth, 337 which was investigated in the current study.

338 In the present study, the swimmers presented significantly lower aBMD and 339 BMAD compared to the impact sports athletes in most skeletal sites and at all three time 340 points (except at baseline at the upper limbs) and in BMD of lower limbs (9-months), WBLH (9-months) and BMAD of WBLH (9-months and 18-months) compared to control group. Interestingly, in a systematic review and meta-analysis by Gomez-Bruton et al [45], analyzed the effect of swimming during childhood and adolescence and found lower

344 values of BMD in swimmers compared to weight-bearing sports. These results 345 corroborate our findings, however, the systematic review also concluded that swimmers 346 show similar values of BMD compared to the control group, unlike the results of our study. Considering that the meta-analysis counted only one longitudinal study, due to the absence of longitudinal studies in the literature, the long-term swimming practice during adolescence may cause lower bone development compared to controls, as found in the present study. Interestingly, the study of Vlachopoulos et al. (2018) [25] found similar results of BMD among swimmers and control group, however, this study was developed with a shorter duration (12 months) and the average weekly training of swimmers was 9.4 hours at baseline while our swimmers practiced 16.6 hours per week. The longer time in training

355 (without impact) could explain the lower values of aBMD and BMAD (WBLH) 356 compared to the control group in our study. Finally, it is important to highlight that the use of bone mineral apparent density (BMAD) allows a more precise analysis of the results, mainly because considers the height of the adolescents, variable which affects comparisons using the areal bone mineral density. 
In our sample, swimmers presented significantly higher values of lean mass than

controls and the literature has emphasized a positive relationship between lean mass and aBMD, even in swimmers $[16,46]$. For this reason, adjustments were made by lean mass in order to specifically analyze the association of sports participation and aBMD/BMAD by removing the effect of lean mass. Thus, the present findings indicate that participation in "hypogravity" environment without gravitational loading, such as swimming, may have negative association with WBLH bone development despite the higher lean mass values [40].

The results of our study were similar with the literature in terms of bone adaptations in skeletal segments (upper and lower limbs) and it highlights the hypothesis that swimming has a non-osteogenic effect in both upper and lower limbs. A recent study by Agostinete et al. [16] focusing on the impact of the training load on the bone health of swimmers, found a negative relationship between lower aBMD at limbs and total body with training load, but not in upper limbs in adolescent swimmers of both sexes. During swimming practice, most of the movements are produced in the horizontal plane, producing forces mainly from the upper limbs, thus reducing the function of the lower limbs only for stabilizing [47]. The forces concentrated in the upper limbs might activate muscle-bone unit to respond differently. Another study developed by Greene et al. [48] in female adolescent water polo athletes strengthens this hypothesis by indicating that there were no significant benefits in bone structure in water polo athletes compared with controls at the lower limbs while in the upper limbs, adolescent female water polo athletes had greater bone strength index at the distal radius compared to non-active girls. The swimming training routine may be related to inhibition of the GH / IGF-1 axis and increased inflammatory markers in the bloodstream generating catabolic responses in bone tissue [49]. 
386 different sports groups. The limitations should also be acknowledged. Firstly, we adopted

387 as inclusion criteria the non-engagement in organized and supervised sports for a

388 minimum period of three months, which is sufficient detraining for significant reductions

389 in physical capacities [50], however, it is plausible that this period should be not sufficient

390 for deficits in exercise's adaptations on BMD. In addition, the minimum sample size was

391 reached only when both sexes are gathered, limiting sex-specific analyses, as well as

392 analysis considering specific sports (impact sports group gathered different sports

393 assuming they have similar mechanical loading [basketball, karate, and judo]). Finally,

394 prolonged exposure in intensive training sessions during growth can be associated with a

395 reduced habitual physical activity practice compared to non-active adolescents as

396 previously analyzed by the literature in swimmers [51], which was not assessed in the 397 present study.

In summary, the present study indicates that children and adolescents engaged in impact sports (basketball and martial arts) accumulate significantly higher aBMD and BMAD compared to children and adolescents engaged in non-impact sports (swimming) over a period of 18 months. Moreover, swimmers had similar values compared to the control group in upper, total spine, and significantly lower limbs, aBMD and BMAD of

403 WBLH compared to the control group. These findings suggest that long-term 404 participation in swimming may not be beneficial for bone health.

405 Most studies in the literature focusing on analysis of different sports modalities 406 on bone health are cross-sectional in design. This cohort study followed children and 407 adolescents for 18 months and indicates that those who were engaged in non-impact 408 sports presented lower aBMD and BMAD accrual compared to those one who were 
engaged in impact sports. These findings hint that it is important of weight-bearing

410 exercises on training routines in order to promote bone health.

411

\section{REFERENCES}

413 1. Pérez-López FR, Chedraui P, Cuadros-López JL (2010) Bone mass gain during 414 puberty and adolescence: deconstructing gender characteristics. Curr Med Chem 17:453-66. doi: 10.2174/092986710790226138

416 2. Baxter-Jones ADG, Faulkner RA, Forwood MR, et al (2011) Bone mineral 417 accrual from 8 to 30 years of age: an estimation of peak bone mass. J Bone Miner $418 \quad$ Res 26:1729-39 . doi: 10.1002/jbmr.412

419 3. Rizzoli R, Bianchi ML, Garabédian M, et al (2010) Maximizing bone mineral 420 mass gain during growth for the prevention of fractures in the adolescents and the 421

4. Smith DM, Nance WE, Kang KW, et al (1973) Genetic factors in determining bone mass. J Clin Invest 52:2800-8 . doi: 10.1172/JCI107476

5. Vasikaran S, Cooper C, Eastell R, et al (2011) International Osteoporosis Foundation and International Federation of Clinical Chemistry and Laboratory Medicine position on bone marker standards in osteoporosis. Clin Chem Lab Med 49:1271-4 . doi: 10.1515/CCLM.2011.602

6. Mouratidou T, Vicente-Rodriguez G, Gracia-Marco L, et al (2013) Associations of Dietary Calcium, Vitamin D, Milk Intakes, and 25-Hydroxyvitamin D With Bone Mass in Spanish Adolescents: The HELENA Study. J Clin Densitom 16:110-117 . doi: 10.1016/j.jocd.2012.07.008

432 7. Vlachopoulos D, Barker AR, Williams CA, et al (2017) The Impact of Sport Participation on Bone Mass and Geometry in Male Adolescents. Med Sci Sports Exerc 49:317-326 . doi: 10.1249/MSS.0000000000001091

435 8. Eisman JA (1999) Genetics of osteoporosis. Endocr Rev 20:788-804 . doi: 10.1210/edrv.20.6.0384

437 9. Kohrt WM, Bloomfield SA, Little KD, et al (2004) American College of Sports 

Exerc 36:1985-96. doi: 10.1249/01.mss.0000142662.21767.58

440 10. Tenforde AS, Fredericson M (2011) Influence of sports participation on bone health in the young athlete: a review of the literature. PM R 3:861-7 . doi:

11. Rauch F, Bailey DA, Baxter-Jones A, et al (2004) The "muscle-bone unit" during the pubertal growth spurt. Bone 34:771-5 . doi: 10.1016/j.bone.2004.01.022

12. Strong WB, Malina RM, Blimkie CJR, et al (2005) Evidence based physical activity for school-age youth. J Pediatr 146:732-7 . doi: 10.1016/j.jpeds.2005.01.055

13. Patel DR, Yamasaki A, Brown K (2017) Epidemiology of sports-related musculoskeletal injuries in young athletes in United States. Transl Pediatr 6:160166 . doi: 10.21037/tp.2017.04.08

14. Ubago-Guisado E, Gómez-Cabello A, Sánchez-Sánchez J, et al (2015) Influence of different sports on bone mass in growing girls. J Sports Sci 33:1710-1718 . doi: 10.1080/02640414.2015.1004639

15. Ubago-Guisado E, García-Unanue J, López-Fernández J, et al (2017) Association of different types of playing surfaces with bone mass in growing girls. J Sports Sci 35:1484-1492 . doi: 10.1080/02640414.2016.1223328

16. Agostinete R, Maillane-Vanegas S, Lynch KR, et al (2017) The impact of training load on bone mineral density of adolescent swimmers: A structural equation modeling approach. Pediatr Exerc Sci 29:520-528 . doi: 10.1123/pes.2017-0008

17. Bielemann RM, Martinez-Mesa J, Gigante DP (2013) Physical activity during life course and bone mass: a systematic review of methods and findings from cohort studies with young adults. BMC Musculoskelet Disord 14:77 . doi: $10.1186 / 1471-2474-14-77$

18. Nikander R, Kannus P, Rantalainen T, et al (2010) Cross-sectional geometry of weight-bearing tibia in female athletes subjected to different exercise loadings. Osteoporos Int 21:1687-94 . doi: 10.1007/s00198-009-1101-0 
19. Zribi A, Zouch M, Chaari H, et al (2014) Enhanced bone mass and physical fitness in prepubescent basketball players. J Clin Densitom 17:156-62 . doi: 10.1016/j.jocd.2013.04.001

20. Ireland A, Maden-Wilkinson T, McPhee J, et al (2013) Upper limb muscle-bone asymmetries and bone adaptation in elite youth tennis players. Med Sci Sports Exerc 45:1749-58 . doi: 10.1249/MSS.0b013e31828f882f

21. Ito IH, Mantovani AM, Agostinete RR, et al (2016) Practice of martial arts and bone mineral density in adolescents of both sexes. Rev Paul Pediatr (English Ed 34:210-215 . doi: 10.1016/j.rppede.2015.09.003

22. Tervo T, Nordström P, Nordström A (2010) Effects of badminton and ice hockey on bone mass in young males: a 12-year follow-up. Bone 47:666-72 . doi: 10.1016/j.bone.2010.06.022

23. Vlachopoulos D, Ubago-Guisado E, Barker AR, et al (2017) Determinants of Bone Outcomes in Adolescent Athletes at Baseline: The PRO-BONE Study. Med Sci Sports Exerc 49:1389-1396 . doi: 10.1249/MSS.0000000000001233

24. Agostinete RR, Lynch KR, Gobbo LA, et al (2016) Basketball Affects Bone Mineral Density Accrual in Boys More Than Swimming and Other Impact Sports: 9-mo Follow-Up. J Clin Densitom 19:375-81 . doi: 10.1016/j.jocd.2016.04.006

25. Vlachopoulos D, Barker AR, Ubago-Guisado E, et al (2018) The effect of 12month participation in osteogenic and non-osteogenic sports on bone development in adolescent male athletes. The PRO-BONE study. J Sci Med Sport 21:404-409 . doi: 10.1016/j.jsams.2017.08.018

26. Zouch M, Vico L, Frere D, et al (2014) Young male soccer players exhibit additional bone mineral acquisition during the peripubertal period: 1-year longitudinal study. Eur J Pediatr 173:53-61 . doi: 10.1007/s00431-013-2115-3

27. Collins AC, Ward KD, McClanahan BS, et al (2019) Bone Accrual in Children and Adolescent Nonelite Swimmers: A 2-Year Longitudinal Study. Clin J Sport Med 29:43-48 . doi: 10.1097/JSM.0000000000000484

28. Behringer M, Gruetzner S, McCourt M, Mester J (2014) Effects of weight- 
498

499

500

501

502

503

504

505

506

507

508

509

510

511

512

513

514

515

516

517

518

519

520

521

522

523

524

525

526

527

bearing activities on bone mineral content and density in children and adolescents: a meta-analysis. J Bone Miner Res 29:467-78 . doi:

29. Zouch M, Zribi A, Alexandre C, et al Soccer increases bone mass in prepubescent boys during growth: a 3-yr longitudinal study. J Clin Densitom 18:179-86 . doi: 10.1016/j.jocd.2014.10.004

30. Katzman DK, Bachrach LK, Carter DR, Marcus R (1991) Clinical and anthropometric correlates of bone mineral acquisition in healthy adolescent girls. J Clin Endocrinol Metab 73:1332-9 . doi: 10.1210/jcem-73-6-1332

31. Maillane-Vanegas S, Agostinete RR, R Lynch K, et al (2018) Bone Mineral Density and Sports Participation. J Clin Densitom (in press). doi: 10.1016/j.jocd.2018.05.041

32. Petit MA, McKay HA, MacKelvie KJ, et al (2002) A randomized school-based jumping intervention confers site and maturity-specific benefits on bone structural properties in girls: a hip structural analysis study. J Bone Miner Res 17:363-72 . doi: 10.1359/jbmr.2002.17.3.363

33. International Society for Clinical Densitometry (2013) Skeletal Health Assessment In Children from Infancy to Adolescence

34. Moreira OC, Oliveira CEP de, De Paz JA (2018) Dual energy X-ray absorptiometry (DXA) reliability and intraobserver reproducibility for segmental body composition measuring. Nutr Hosp 35:340-345 . doi: 10.20960/nh.1295

35. GE Healthcare Lunar (2010) enCORE-Based X-ray Bone Densitometer: User Manual

36. Moore SA, McKay HA, Macdonald H, et al (2015) Enhancing a somatic maturity prediction model. Med Sci Sports Exerc 47:1755-1764 . doi: 10.1249/MSS.0000000000000588

37. Wilkinson K, Vlachopoulos D, Klentrou P, et al (2017) Soft tissues, areal bone mineral density and hip geometry estimates in active young boys: the PROBONE study. Eur J Appl Physiol 117:833-842 . doi: 10.1007/s00421-017-3568-2

38. Seeman E (2002) An exercise in geometry. J Bone Miner Res 17:373-80 . doi: 
529 39. Hind K, Burrows M (2007) Weight-bearing exercise and bone mineral accrual in children and adolescents: a review of controlled trials. Bone 40:14-27 . doi: 10.1016/j.bone.2006.07.006

532

40. Ferry B, Duclos M, Burt L, et al (2011) Bone geometry and strength adaptations to physical constraints inherent in different sports: Comparison between elite female soccer players and swimmers. J Bone Miner Metab 29:342-351 . doi: 10.1007/s00774-010-0226-8

41. Dias Quiterio AL, Carnero EA, Baptista FM, Sardinha LB (2011) Skeletal mass in adolescent male athletes and nonathletes: relationships with high-impact sports. J strength Cond Res 25:3439-47 . doi: 10.1519/JSC.0b013e318216003b

42. Ito IH, Kemper HCG, Agostinete RR, et al (2017) Impact of Martial Arts (Judo, Karate, and Kung Fu) on Bone Mineral Density Gains in Adolescents of Both Genders: 9-Month Follow-Up. Pediatr Exerc Sci 29:496-503 . doi: 10.1123/pes.2017-0019

43. Duckham RL, Baxter-Jones ADG, Johnston JD, et al (2014) Does physical activity in adolescence have site-specific and sex-specific benefits on young adult bone size, content, and estimated strength? J Bone Miner Res 29:479-86 . doi: 10.1002/jbmr.2055

44. Vlachopoulos D, Barker AR, Ubago-Guisado E, et al (2017) Longitudinal Adaptations of Bone Mass, Geometry, and Metabolism in Adolescent Male Athletes: The PRO-BONE Study. J Bone Miner Res 32:2269-2277 . doi: 10.1002/jbmr.3206

45. Gomez-Bruton A, Montero-Marín J, González-Agüero A, et al (2016) The Effect of Swimming During Childhood and Adolescence on Bone Mineral Density: A Systematic Review and Meta-Analysis. Sports Med 46:365-79 . doi: $10.1007 / \mathrm{s} 40279-015-0427-3$

46. Ubago-Guisado E, Vlachopoulos D, Ferreira de Moraes AC, et al (2017) Lean mass explains the association between muscular fitness and bone outcomes in 13year-old boys. Acta Paediatr 106:1658-1665 . doi: 10.1111/apa.13972 
558 47. Gourgoulis V, Boli A, Aggeloussis N, et al (2014) The effect of leg kick on

559

560

561

562

563

564

565

566

567

568

569

570

571

572

573

574 sprint front crawl swimming. J Sports Sci 32:278-89 . doi: $10.1080 / 02640414.2013 .823224$

48. Greene DA, Naughton GA, Bradshaw E, et al (2012) Mechanical loading with or without weight-bearing activity: influence on bone strength index in elite female adolescent athletes engaged in water polo, gymnastics, and track-and-field. J Bone Miner Metab 30:580-7 . doi: 10.1007/s00774-012-0360-6

49. Nemet D, Oh Y, Kim H-S, et al (2002) Effect of intense exercise on inflammatory cytokines and growth mediators in adolescent boys. Pediatrics 110:681-689 . doi: 10.1542/peds.110.4.681

50. Ormsbee MJ, Arciero PJ (2012) Detraining increases body fat and weight and decreases VO2peak and metabolic rate. J strength Cond Res 26:2087-95 . doi: 10.1519/JSC.0b013e31823b874c

51. Gómez-Bruton A, González-Agüero A, Gómez-Cabello A, et al (2016) Swimming and bone: Is low bone mass due to hypogravity alone or does other physical activity influence it? Osteoporos Int 27:1785-93 . doi: 10.1007/s00198015-3448-8 
Table 1. Descriptive characteristics of the sample stratified by sports at baseline $(n=71)$

\begin{tabular}{|c|c|c|c|c|}
\hline & $\begin{array}{c}\text { Control Group } \\
(\mathrm{n}=20,12 \text { males }) \\
\text { Mean (SD) }\end{array}$ & $\begin{array}{c}\text { Non-Impact sport } \\
\text { (n=18, } 11 \text { males) } \\
\text { Mean (SD) }\end{array}$ & $\begin{array}{c}\text { Impact Sports } \\
(\mathrm{n}=33,19 \text { males }) \\
\text { Mean }(\mathrm{SD})\end{array}$ & p-value \\
\hline Chronological age (years) & $12.6(2.6)$ & $12.7(1.2)$ & $12.7(1.3)$ & 0.955 \\
\hline Body mass (kg) & $46.6(12.4)$ & $53.7(11.2)$ & $53.7(14.7)$ & 0.134 \\
\hline Height $(\mathrm{cm})$ & $153.9(11.6)$ & $164.0(10.5)^{\mathrm{a}}$ & $159.8(13.9)$ & 0.049 \\
\hline Years from PHV (years) & $-2.7(1.2)$ & $-1.9(1.7)$ & $-2.3(1.6)$ & 0.290 \\
\hline APHV (years) & $15.4(2.1)$ & $14.8(1.3)$ & $15.0(1.2)$ & 0.459 \\
\hline Lean soft tissue (kg) & $30.3(6.7)$ & $40.8(8.6)^{\mathrm{a}}$ & $37.5(9.7)^{\mathrm{a}}$ & 0.001 \\
\hline Fat mass $(\mathrm{kg})$ & $13.2(7.4)$ & $9.8(4.8)$ & $14.1(9.8)$ & 0.197 \\
\hline Fat mass $(\%)$ & $27.2(10.0)$ & $18.1(7.3)^{\mathrm{a}}$ & $23.4(9.7)$ & 0.014 \\
\hline fat mass of trunk $(\%)$ & $28.5(10.7)$ & $19.9(8.0)^{\mathrm{a}}$ & $25.4(10.6)$ & 0.032 \\
\hline \multicolumn{5}{|l|}{$\begin{array}{l}\text { Areal bone mineral density } \\
\left(\mathrm{g} / \mathrm{cm}^{2}\right)\end{array}$} \\
\hline Upper limbs & $0.685(0.077)$ & $0.750(0.071)$ & $0.766(0.142)^{\mathrm{a}}$ & 0.039 \\
\hline Lower limbs & $1.085(0.145)$ & $1.115(0.134)$ & $1.197(0.142)^{\mathrm{a}}$ & 0.016 \\
\hline Total spine & $0.894(0.184)$ & $0.957(0.106)$ & $0.996(0.138)$ & 0.055 \\
\hline WBLH & $0.915(0.118)$ & $0.953(0.094)$ & $1.003(0.115)^{\mathrm{a}}$ & 0.023 \\
\hline BMAD-WBLH & $0.091(0.008)$ & $0.085(0.004)^{\mathrm{a}}$ & $0.090(0.007)^{\mathrm{b}}$ & 0.016 \\
\hline \multicolumn{5}{|l|}{ Training parameters } \\
\hline Weekly training (min/week) & -- & $1001(196)$ & $664(369)^{\mathrm{b}}$ & 0.001 \\
\hline Sporting experience (months) & -- & $56.2(38.9)$ & $39.9(31.2)$ & 0.139 \\
\hline RT (number athletes) & -- & 11 & 13 & - \\
\hline
\end{tabular}

Significant differences among the groups (ANOVA) in bold. $a=$ denotes significant difference compared to Control; $b=$ denotes significant different compared to Swimmers; SD (standard deviation); PHV= peak height velocity; APHV= age of peak height velocity; $\mathrm{RT}=$ resistance training; $\mathrm{BMAD}=$ Bone mineral apparent density; WBLH=Whole body less head. 


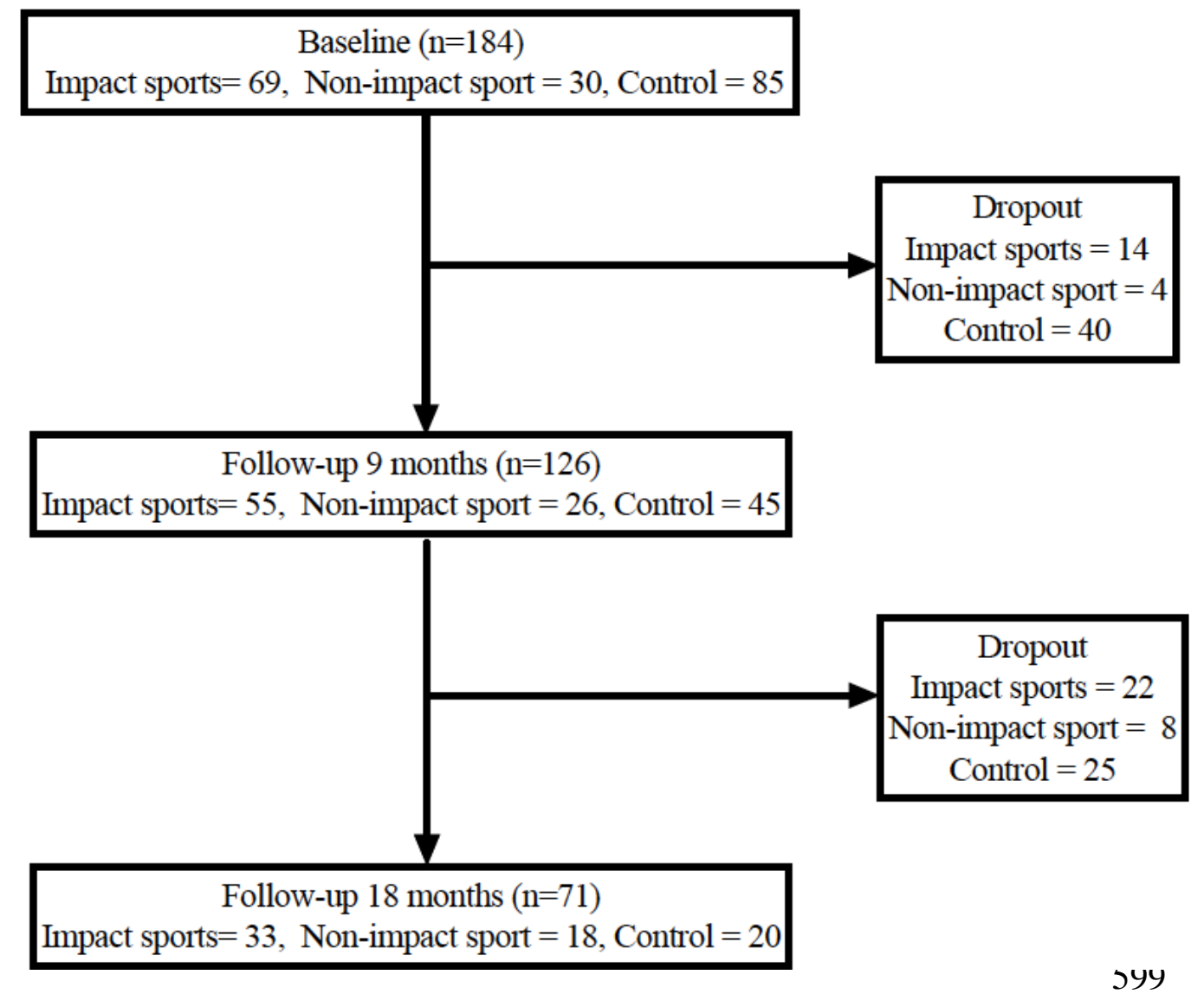

600 Figure 1. Flowchart of the study design 
A

aBMD-Upper Limbs

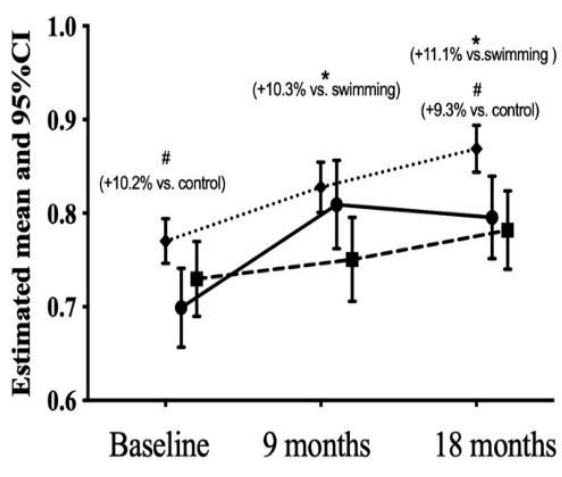

C

aBMD-Spine

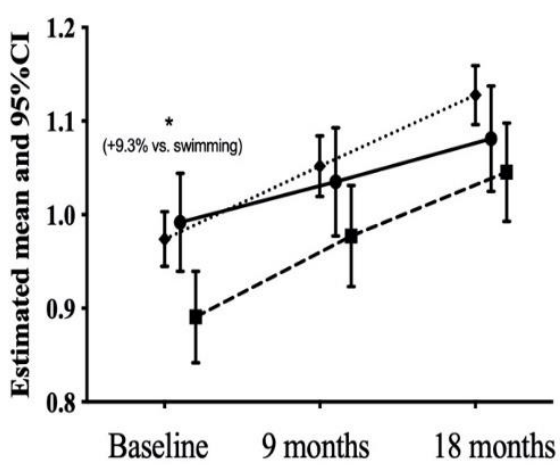

B

aBMD-Lower Limbs

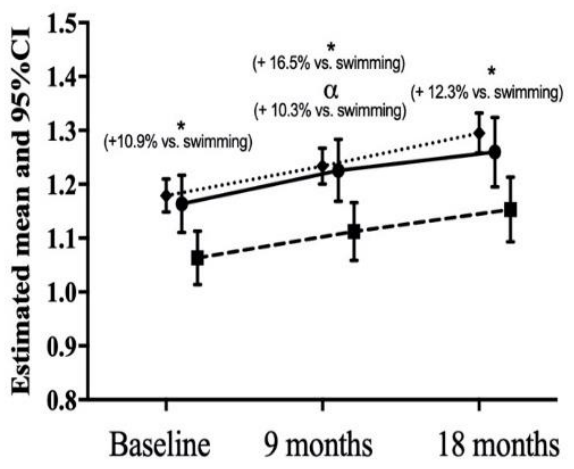

D

aBMD-Whole Body

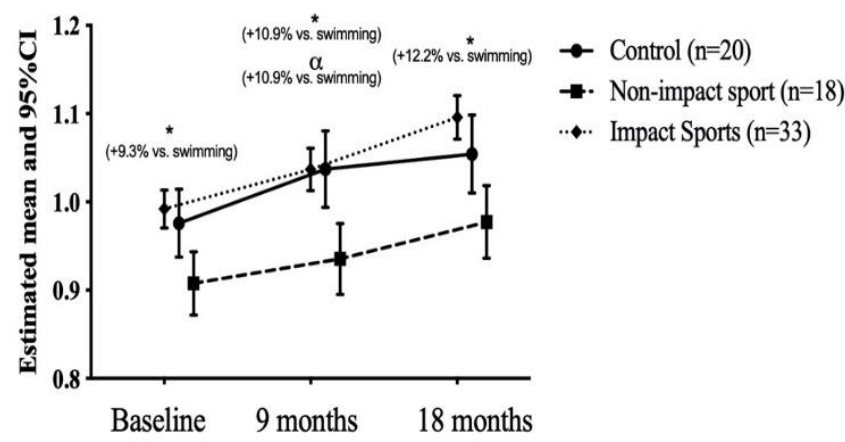

Figure 2. Areal bone mineral density (aBMD, $\left.\mathrm{g} / \mathrm{cm}^{2}\right)$ status among adolescents of impact sports, non603 impact sport and control group adjusted by age of PHV, lean soft tissue, volume of training, practice of 604 resistance training, sporting experience, fat mass, height, vitamin D intake and sex. *significant difference 605 between non-impact and impact sports; ${ }^{\alpha}$ significant difference between non-impact and control group; 606 "significant difference between impact sports and control group. Values between brackets represent the 607 percentage difference between the groups. 


\section{BMAD-Whole Body}

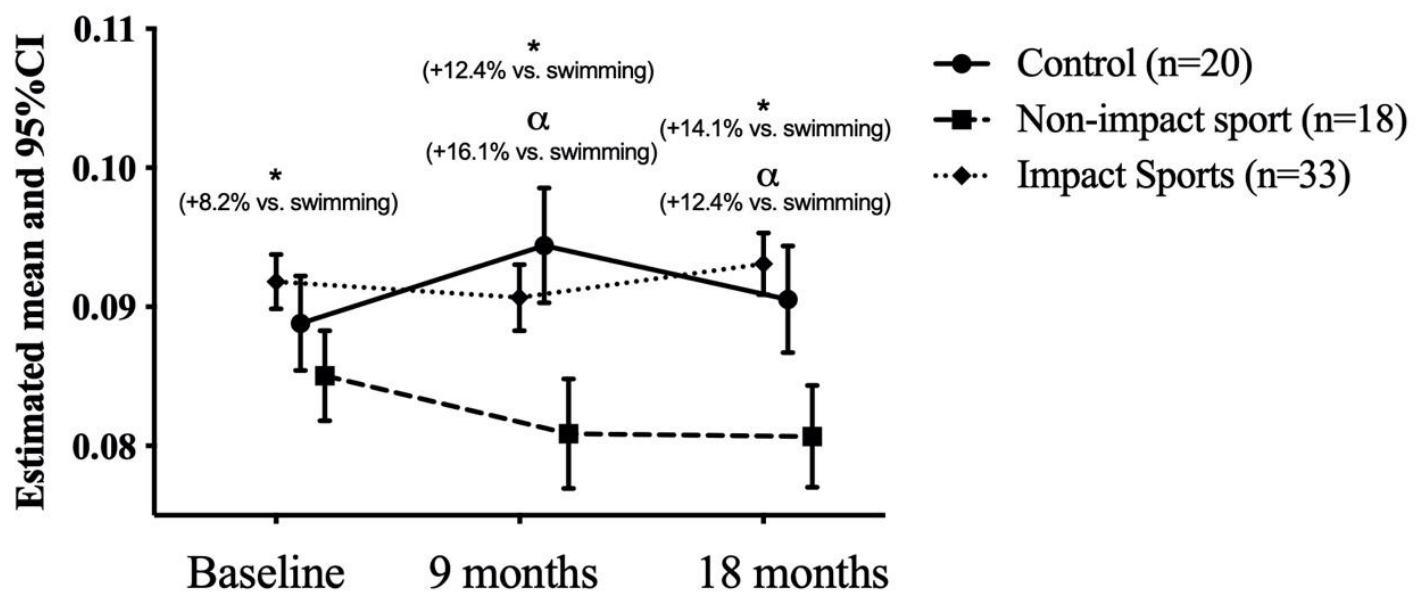

609 Figure 3. Bone mineral apparent density $\left(, \mathrm{g} / \mathrm{cm}^{3}\right)$ status among adolescents of impact sports, non-impact 610 sport and control group adjusted by age of PHV, lean soft tissue, volume of training, practice of resistance 611 training, sporting experience, fat mass, height, vitamin D intake and sex. *significant difference between 612 non-impact and impact sports; ${ }^{\alpha}$ significant difference between non-impact and control group. Values 613 between brackets represent the percentage difference between the groups. 